\title{
EchoGéo
}

29 | 2014

The Political Ecology of Conservation and

Development Territories

\section{Les langues de la diffusion scientifique : une question pour les géographes et les géographies}

Nathalie Lemarchand and Antoine Le Blanc

\section{(2) OpenEdition}

Journals

Electronic version

URL: https://journals.openedition.org/echogeo/13941

DOI: 10.4000/echogeo.13941

ISSN: 1963-1197

Publisher

Pôle de recherche pour l'organisation et la diffusion de l'information géographique (CNRS UMR 8586)

Electronic reference

Nathalie Lemarchand and Antoine Le Blanc, "Les langues de la diffusion scientifique : une question pour les géographes et les géographies", EchoGéo [Online], 29 | 2014, Online since 03 October 2014 connection on 01 August 2021. URL: http://journals.openedition.org/echogeo/13941 ; DOl: https:// doi.org/10.4000/echogeo.13941

This text was automatically generated on 1 August 2021

EchoGéo est mis à disposition selon les termes de la licence Creative Commons Attribution - Pas d'Utilisation Commerciale - Pas de Modification 4.0 International (CC BY-NC-ND) 


\title{
Les langues de la diffusion scientifique : une question pour les géographes et les géographies
}

\author{
Nathalie Lemarchand and Antoine Le Blanc
}

1 Le Comité National Français de Géographie ${ }^{1}$ a organisé un forum sur le rapport entre la langue et les contextes sociétaux de production de la connaissance et, plus largement, la langue internationale de l'échange scientifique: l'anglais. Étaient invités les géographes francophones et francophiles, de contextes nationaux et linguistiques variés, mais aussi tous les géographes intéressés à partager leur expérience de la production et de la diffusion du savoir dans la mondialisation. Le forum s'est tenu les 3 et 4 juillet 2014 à Paris, dans les locaux de la Maison de la Recherche de la Sorbonne, et a réuni enseignants-chercheurs et chercheurs, directeurs de revue, doctorants, personnels institutionnels. Plusieurs nationalités étaient représentées (Belgique, Canada, Royaume-Uni, Allemagne, Russie...), les personnes présentes ayant toutes des expériences internationales variées. Le Président de l'Union Géographique Internationale, Vladimir Kolossov, ainsi que Dieter Soyez, vice-président de l'UGI, ont pris part aux différents ateliers du forum. Les débats ont eu lieu en français, sans s'interdire des excursions dans d'autres langues.

Le forum répondait à un questionnement multiple concernant la place des langues dans la production et la diffusion scientifiques. Il importe en effet de se questionner sur les effets que la « globalisation » a sur la production et la diffusion du savoir géographique. Comme c'est le cas dans de très nombreux pays, les géographes français sont plus que jamais soumis à la pression de publier en anglais, pression qui se répercute sur les choix des revues scientifiques et les autres supports de publication. Cette situation est souvent appréhendée avec fatalisme, sinon comme une nécessité afin de faire connaître ses travaux à l'échelle internationale. Mais n'y a-t-il pas quelque chose qui se perd avec la traduction? Plus important peut-être, y a-t-il quelque chose qui se perd avec une production scientifique en sciences sociales réduite à une seule langue globale d'expression? Ce questionnement va plus loin que la problématique du déclin du 
français comme langue internationale, il s'agit plutôt de se pencher sur la production du savoir telle qu'elle se réalise par le processus en cours de normalisation par l'anglais scientifique. Que devient la diversité des démarches dans une science globale? Qu'en est-il de la valeur des études locales ? Le débat peut être élargi à la formation en anglais et à une internationalisation dont les conséquences scientifiques sont peu étudiées pour le moment.

3 Lors du forum, les débats ont eu lieu sous forme d'ateliers qui distinguaient quatre grandes thématiques :

- Globalisation/mondialisation du savoir: quels sont les modes de production et de diffusion d'un savoir globalisé ? Quelle place et quelle valeur accorder à la traduction? Comment appréhender l'évolution de la science géographique vers une production scientifique plus standardisée?

- Production scientifique et contexte «local» (sociétal, national, linguistique) : quelle place pour la diversité des contextes « locaux » dans la production scientifique mondialisée? Quelle est la responsabilité des chercheurs et chercheuses dans la production de ce savoir globalisé, et dans le maintien d'un lien avec la société civile locale ? Comment appréhender la place croissante des revues et des normes qu'elles véhiculent, notamment sur le plan linguistique, avec les conséquences que cela implique pour la géographie et l'aménagement?

- Diffusion du savoir : quels sont les moyens d'expression et les réseaux d'une collectivité géographique scientifique particulière et sa validation scientifique aux échelles locale et internationale? Quelle place pour des leviers de diffusion, nouveaux ou plus anciens, mais moins mis en avant que les articles de revues, comme les livres, les films ou les jeux vidéo? Quel rôle peut jouer la géographie par le biais de la cartographie?

- Formation : quels liens entre la publication des travaux scientifiques des géographes et la formation supérieure? Comment encadrer ou appréhender le développement de l'enseignement en anglais ou dans d'autres langues? Quel rôle peut jouer la géographie dans la géopolitique mondiale de l'éducation et de la formation?

4 Le forum a permis de dégager un certain nombre de pistes, voire de préconisations, au sujet de l'internationalisation de la production et de la diffusion scientifique, concernant $\mathrm{du}$ moins la géographie française et francophone. Le forum et ses propositions ont été présentés lors du Congrès de l'UGI à Cracovie en août 2014, et le rapport final sera publié dans une revue internationale, diffusé auprès de l'ensemble des géographes francophones et de l'UGI, envoyé aux instances institutionnelles françaises et européennes de la recherche et de l'enseignement supérieur. Il s'agit, constat partagé par tous, de rappeler l'importance du multilinguisme et de prendre en compte les spécificités de la science géographique dans la mondialisation: la géographie, en tant que science des territoires et de leurs mutations, entretient des interactions complexes avec la mondialisation, chacune façonnant l'autre de manière continue, chacune étant à la fois miroir et facteur de modification de l'autre.

5 En effet, les conclusions et propositions du forum visent essentiellement à favoriser le multilinguisme, sans être dans une posture d'opposition à l'anglais, tout au contraire : il faut viser à créer des espaces de cohabitation des langues, valoriser les traductions (dans les CV par exemple), encourager le multilinguisme dans les références... Citons également, parmi les propositions, le projet de mettre en place une plateforme internationale de traduction d'articles, voire une revue qui aurait ce rôle. Les institutions publiques et privées peuvent être mobilisées, les financements constituant 
des leviers puissants pour favoriser le multilinguisme, par exemple en privilégiant des langues locales, ou en assurant la diffusion multilingue d'expérimentations locales.

6 Les spécificités de la géographie sont nombreuses et peuvent constituer des atouts dans l'internationalisation scientifique, tout en ne réduisant pas la diffusion scientifique à un plus petit dénominateur linguistique commun; le lien avec l'aménagement local nécessite l'utilisation de langues locales, par exemple. Les langages cartographiques constituent également une opportunité d'aller vers une internationalisation "positive ", qui ne serait pas un nivellement a minima de la diffusion scientifique, de même que d'autres leviers de diffusion que les revues (ouvrages, MOOC, films, jeux vidéo...).

7 Pour la recherche géographique francophone en particulier, plusieurs questions ont été posées, et diverses pistes ont été soulevées, dans l'objectif de mobiliser les géographes francophones non français, par le biais, par exemple, d'un réseau, d'associations ou de financements.

8 Enfin, la diversité des parties prenantes doit être valorisée et mise en œuvre dans différents domaines et différentes structures, comme dans les comités de rédaction des revues, ou dans les systèmes de recrutement des enseignants et chercheurs, qui doivent être plus ouverts.

9 Toutes ces propositions n'ont pas la même facilité de mise en œuvre : c'est pourquoi il s'agissait ici d'une première étape d'un processus. Le Comité National Français de Géographie pourra accompagner ou mettre en œuvre certaines de ces préconisations, mais, pour d'autres, ce sont de multiples autres acteurs qui sont concernés. L'internationalisation de la production et de la diffusion scientifiques en géographie est une chance, à condition qu'elle soit accompagnée et voulue, et non subie.

\section{NOTES}

1. Créé à l'initiative de l'Académie des Sciences, le CNFG est membre du Comité Français des Unions Scientifiques Internationales (COFUSI). À l'échelle nationale, le CNFG constitue l'une des instances importantes de la recherche scientifique, à côté du Comité National du CNRS et des commissions scientifiques de l'IRD. Le CNFG gère des commissions au nombre de 23. À l'échelle internationale, il est membre de l'Union Géographique Internationale. Entre autres missions, le CNFG propose des orientations pour la recherche et l'enseignement de la géographie en France, par le biais de ses commissions thématiques ou par l'organisation d'événements de portée nationale ou internationale (www.cnfg.fr/). 


\section{ABSTRACTS}

In July 2014, the CNFG (French National Committee for Geographers) organized a two-day forum to discuss the relationship between languages, social contexts of scientific production, and the overtly admitted language of scientific exchange, that is, English. It invited all French-speaking geographers, from different "national" and "linguistic" contexts, but also all geographers and geographies interested in sharing their views and experience on the diffusion and production of knowledge in the global context. Indeed, globalization affects both the production and diffusion of scientific knowledge in all regions of the World, where English has become the de facto lingua franca of science. As it is the case in many countries, French geographers are more than ever under pressure to publish in English, and so are their scientific journals. This is often talked about as a fatality or else, as a necessity, in order to make one's work known in world geographic circles. But is there something lost in translation? More importantly, is there something lost in the production of scientific work in the social sciences within a single global language? Those questions go far beyond the simple question of the decline of French as an international language; it raises crucial interrogations such as the normalizing process of "scientific English" in the social sciences, the input of diversity in the global science, the disqualification of "local" case studies, etc. At this Forum, many countries were represented (Belgium, Canada, UK, Germany, Russia ...), which expressed a diverse International experience. The IGU President Vladimir Kolosov and Dieter Soyez of the UGI Executive Board participated. Four round tables were organized: Globalization of knowledge; Global science and the local; Knowledge dissemination; Academic Formation. The account of the Forum and the ideas and proposals that came out of it have been presented at the Congress of the IGU in Krakow in August 2014. The final report will be published in an International journal.

\section{AUTHORS}

\section{NATHALIE LEMARCHAND}

Nathalie Lemarchand, géographe, nlemarchan@aol.com, est Professeur d'université, à l'Université de Paris 8, membre de LADYSS UMR 7533. Elle est, avec Antoine Le Blanc, viceprésidente du Comité National Français de Géographie en charge des manifestations et des relations internationales.

\section{ANTOINE LE BLANC}

Antoine Le Blanc, géographe, Antoine.Le-Blanc@univ-littoral.fr, est Maître de conférences à l'Université du Littoral, membre de TVES EA 4477. Il est, avec Nathalie Lemarchand, viceprésident du Comité National Français de Géographie en charge des manifestations et des relations internationales. 